\title{
Digital Transformation of Business Processes of an Enterprise
}

\author{
Viktoriia Kondarevych ${ }^{1}$, Kateryna Andriushchenko ${ }^{1}$, Nataliia Pokotylska ${ }^{2}$, \\ Ganna Ortina ${ }^{3}$, Olga Zborovska ${ }^{4}$, Lyubov Budnyak ${ }^{5}$ \\ ${ }^{1}$ SHEE «Kyiv National Economic University named after Vadym Hetman», Department of Economics and \\ Entrepreneurship, Peremogy ave., 54/1, Kyiv, , 03057, Ukraine \\ ${ }^{2}$ State Agrarian and Engineering University in Podilia, Shevchenka str., 13, \\ Kamianets-Podilskyi, 32300,Ukraine \\ ${ }^{3}$ Dmytro Motornyi Tavria State Agrotechnological University, Department of Public Administration, \\ Administration and Law, 18 B.Khmelnytsky Ave, Melitopol, Zaporizhzhia obl., 72310, Ukraine \\ ${ }^{4}$ Department of finance, banking and insurance of National University in Zaporizhzhya, Zhukovsky str., 66, \\ Zaporizhzhya, 69600, Ukraine \\ ${ }^{5}$ State Agrarian and Engineering University in Podilia, Department of Finance, Banking, Insurance and \\ Electronic Payment Systems, Shevchenka str., 13, Kamianets-Podilskyi, 32300, Ukraine
}

\begin{abstract}
Industry 4.0 is the digital transformation of business processes. In the process of modernization of the enterprise in question, a new production factor is gradually replacing the previous production factor. At the same time, the value of the output of the old production decreases to zero, and the limit value of the output of the new production is steadily increasing. The production functions for each production component and the enterprise as a whole, based on the results of a numerical solution of the Cauchy problem, which describe the process of replacing one production enterprise with another, are proposed.
\end{abstract}

Keywords - digital transformation, business processes, smart product.

DOI: 10.18421/TEM94-63

https://doi.org/10.18421/TEM94-63

Corresponding author: Viktoriia Kondarevych, PhD-student of Department of Economics and Entrepreneurship SHEE "Kyiv National Economic University named after Vadym Hetman", Peremogy ave., 54/1, Kyiv, Ukraine, 03057.

Email: kondarevychv@gmail.com

Received: 18 August 2020.

Revised: 09 November 2020.

Accepted: 16 November 2020.

Published: 27 November 2020.

(cc) BY-NC-ND (C) 2020 Viktoriia Kondarevych et al; published by UIKTEN. This work is licensed under the Creative Commons Attribution-NonCommercial-NoDerivs 4.0 License.

The article is published with Open Access at www.temjournal.com

\section{Introduction}

A recent survey made by the University of North Carolina found that in 2019 directors of companies have moved away from digital euphoria and see digital transformation (DT) as the main risk factor, although last year it occupied only 10th place. The growing concern is that universal recipes for transformation do not exist and, as a result, $70 \%$ of the "digital" initiatives that companies are trying to implement do not reach their goals. This means that $\$ 1.3$ trillion that was spent on DT last year, it was estimated that $\$ 900$ billion went to waste.

In successful organizations, the introduction of automation technology begins with an analysis of the ways the employees work. Then using digital technology, the results are put into practice. The ability to extract information from text, images and other unstructured data can be compared with the work of the human eye, and the ability to correlate the characteristics of repetitive tasks and perform operations that require specialized knowledge, with the work of the central nervous system, which simultaneously processes various kinds of signals.

However, the digital transformation of business processes is not limited only to the tasks of each individual employee, but covers a huge amount of different types of activities and processes within the enterprise. Digitalization of the operational business processes of each type of work will help the company achieve better results.

Choosing an approach to digital transformation within business processes, two main characteristics have to be taken into account: the volume and the uniqueness of the work. The first group includes repetitive tasks performed in large volumes: these 
tasks are performed uniformly several thousand, or even hundreds of thousands times a day. The second group consists of unique single tasks: the number of such tasks is much smaller, and their implementation may require non-standard approaches.

By combining various technologies, enterprises receive tools that allow them to increase the output of products, reduce the level of rejects, reduce the consumption of materials, and increase the availability of equipment (Fig. 1).

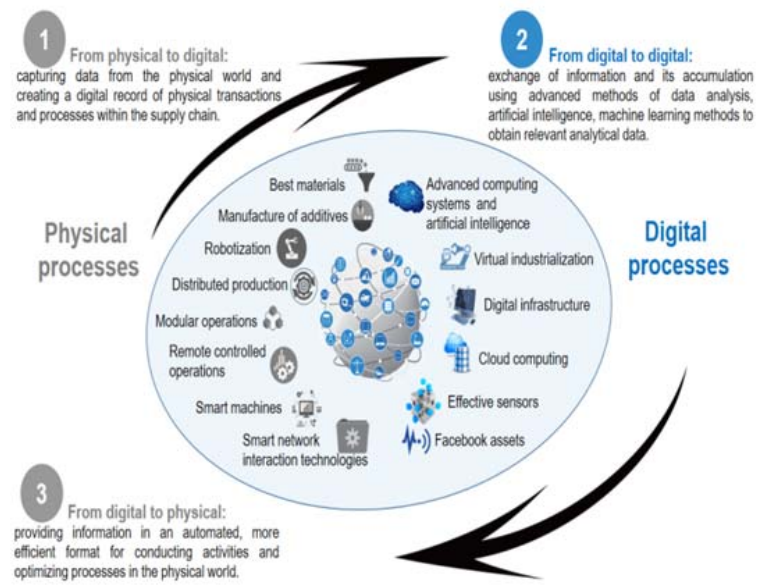

Figure 1. "Smart" connection - data circulation as a part of the digitalization of business processes in the supply chain

If we take these two characteristics as coordinate axes, we get a diagram of the types of activities depending on the volume and uniqueness of the tasks performed in the organization. The benefits provided by the digital transformation of business processes depend on the type of activity (Fig. 2).

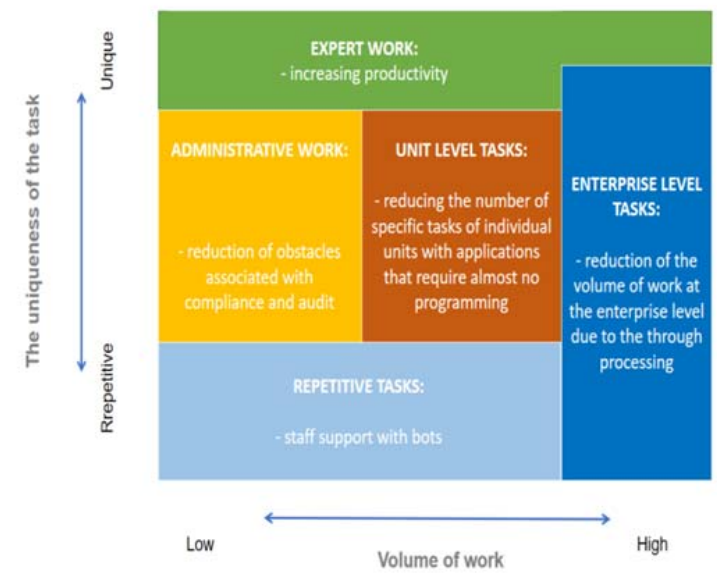

Figure 2. Digitalization of available business processes of all types of enterprise activities

- Repetitive tasks - this type of activity provides for a very insignificant or even zero subjective assessment, does not require special skills, but plays a key role in doing business. Routine tasks such as processing invoices and payments or making standard decisions (for example, identifying additional agreements to an insurance contract) can be digitalized using bots that help empower employees and allow them to focus on other tasks.

- Administrative tasks - these tasks belong to the middle range they are moderately unique and not numerous. These include everyday routine tasks such as viewing and approving documents and policies - processes that can be improved through automation. In addition, we can digitalize components of other administrative tasks, such as enforcing policies to ensure regulatory compliance.

- Enterprise level tasks - this group includes different tasks both in terms of the volume and the uniqueness of the work performed; these tasks span multiple units within the organization. Operations such as approving loan applications involving various specialists in an enterprise can also have a significant impact on profits. However, with the growth of uniqueness, the complexity and costs of performing such operations manually increase. Digital transformation of business processes would accelerate processing and increase profits.

- Expert work - this category includes the most unique and important tasks performed by experts. But even highly skilled work can be improved with digitalization, increasing productivity through faster and more scalable data collection and interpretation.

\section{Literature Survey}

Brynjolfsson and McAfee (2014) in their book talk about a new stage in business development, which is characterized by innovation [4]. Digital transformation of the enterprise's business processes is able to replace people as much as possible with machines based on "artificial intelligence". In this regard, Westerman et al. (2014) in their article describe the cyclical nature of technological innovations that affect the development of a business and its transformation [19]. The authors focus on the industrial revolution when it is based on the introduction of new machines. Vogelsang (2010) emphasizes that there were not many who wanted to introduce IT innovations into the business processes of their companies, the ones that did it were the first to pave the way for technological innovations, which created the time of digitalization [18]. It should be noted that the term "digital divide" arose in connection with the big increase in the introduction of information technology in the 1990s. In recent years, this term has been used to describe differences in information inequality [21]. Frank et al. (2014) discusses that society is at the intersection of the 
fourth and fifth technological revolution. The fifth revolution (or as it is called in the literature "wave") already affects social, mobile, analytical and cloud (hereinafter SMAC) technologies. It is predicted that the number of companies that will digitalize business processes will increase approximately tenfold with each next technological revolution, which will lead to the use of at least 25 billion digital tools by 2025 [8]. Many articles to date suggest that SMAC technologies are the most influential tools for digital transformation of business processes [7], [17].

At the macro level, affirm that the digital transformation of business processes is basically a complex organizational process. But in turn, it is noted that digital transformation of business processes offers a number of advantages: a stronger experimental potential, more dispersed technological innovations, a higher level of formation of innovative business processes [15], [16].

At the micro level - one can consider the digital transformation of business processes through implementation: Big Data Analytics, AI, 5G, blockchain, Internet of things, digital platforms [6], [15]. As it already has been mentioned in several studies, the digital transformation of business processes (the introduction of digital innovations and technologies) can change and unite industries, and become intersectional [3], [14], and [20]. Besides, the digital transformation of business processes has the potential to bring together users and industries, reprogramming products and, most importantly, to digitize non-digital products and services. This is especially true for industrial enterprises that produce analog products [18]. Companies that, regardless of size, focused on technological development and digitalization of business processes, were able to quickly adapt to changes in the external environment, become market leaders, develop intersectoral cooperation [5], [14].

Uber, the world's largest taxi service organization, has no vehicles, and Airbnb, the world's largest nonreal estate provider. In the same way, companies such as Facebook, Google or Amazon created entire business processes based on digital innovation and digitalization of their business models. Unlike Dell, Nokia or Kodak underestimated innovative technologies and changes in the external environment [12] refused to digitalize business processes, which led to the loss of the market. The general understanding of digitalization has shifted from the introduction of computer equipment in the enterprise to the digital transformation of business processes (B2B) and the orientation of the business client (B2C), focusing on circumstances [13]. Companies that are digitalizing their business processes are facing a new paradigm of risk management [10].
Today, most companies around the world are in the process of transitioning from automation of operations to digital transformation of business processes as the formation of a new form of fixed assets. The main reason for the relevance of digital transformation of business processes in the enterprise is associated with the rapid development of cloud computing. This leads companies and private users to own technologies, such as operating systems, applications, cloud servers, in order to be an active user. The digital transformation of business processes is seen as an asset based on technological modernization that affects organizational structures, strategies and business processes [1]. However, Baharun et al. argue that the increased amount of data that we use in our companies transforms the availability of information, thereby increasing the overall performance of the company [2].

\section{Methods}

We can consider a manufacturing enterprise whose capacities provide output by one resource in the form of a certain volume of the production factor. If the introduction of digital tools for the modernization of technological equipment takyi place at this enterprise, then a new digital production appears and develops within the existing production. Both of these productions work in parallel for a while, until the new production completely displaces the old production.

The old production of the enterprise spends a certain set of resources in the form of the volume of the factor of production $Q$, and the new production of the enterprise spends its set of resources in the form of the volume of the factor of production $P$. These factors of production $Q$ and $P$ are composed of fixed capital, production assets involved in the production of labor resources used in the production of materials, applied technologies, various kinds of innovations, etc.

The time variable $t$ is assumed to be continuous, the unit of measurement is the so-called production period (month, quarter, year). The functions $Q=Q$ (t) and $P=P(t)$ are assumed to be continuous, continuously differentiable, and bounded on the numerical axis $(0<t<\infty)$

$$
\begin{gathered}
Q_{0}<Q(t)<Q_{\infty}, \\
P_{0}<P(t)<P_{\infty} . \\
Q_{0}=\lim _{t \rightarrow 0} Q(t), Q_{\infty}=\lim _{t \rightarrow \infty} Q(t), \\
P_{0}=\lim _{t \rightarrow 0} P(t), P_{\infty}=\lim _{t \rightarrow \infty} P(t) .
\end{gathered}
$$

The initial values of the production factors $Q_{0}$ and $P_{0}$ are considered known, the limiting values of $Q_{\infty}$ and $P_{\infty}$ are to be calculated. 
Product releases from the old production facility at $Q V$ and the new production facility at $P V$ are provided by the two Cobb-Douglas manufacturing functions.

$$
V_{Q}=R_{Q} \cdot Q^{a_{Q}}, V_{P}=R_{P} \cdot P^{a_{P}}
$$

Here, $\mathrm{R}_{Q} R_{P}$ is the cost of products produced per unit volume of resources, power-law indicators of production functions, $a_{Q} a_{P}$ are the elasticities of output for the corresponding resources, with the inequalities $0<a_{Q}<1,0<a_{P}<1$.

To assess the dynamics of development of the considered production enterprises, it is necessary to draw up balance equations for the volumes of production factors $Q$ and $P$.

The values of changes in the volumes of production factors $\Delta Q$ and $\Delta P$ for a certain small period of time $\Delta \mathrm{t}$ can be represented by the relations

$$
\begin{aligned}
& \Delta Q(t)=\Delta Q^{A}(t)+\Delta Q^{I}(t)+\Delta Q^{G}(t), \\
& \Delta P(t)=\Delta P^{A}(t)+\Delta P^{I}(t)+\Delta P^{G}(t) .
\end{aligned}
$$

Here $\triangle Q A(t), \triangle P A(t)$ are partial depreciation of production factors, $\triangle Q I(t), \triangle P I(t)$ are partial recovery of production factors due to internal endogenous investments in the enterprise in question, $\triangle Q G(t), \triangle P G(t)$ - partial recovery of production factors due to external exogenous investments in the enterprise in question.

Here we restrict ourselves to the case of proportional partial depreciation $\triangle Q A(t), \triangle P A(t)$ for the time interval $\Delta t$

$$
\begin{aligned}
& \Delta Q^{A}(t)=-A_{Q} \cdot \theta(t) \cdot Q(t) \cdot \Delta t, \\
& \Delta P^{A}(t)=-A_{P} \cdot \theta(t) \cdot P(t) \cdot \Delta t .
\end{aligned}
$$

Here, $A_{Q} A_{P}$ - depreciation ratios, the share of volumes of factors of production disposed of per unit time.

The increments of internal endogenous investments $\Delta Q^{I}(t), \Delta P^{I}(t)$ for the period of time $\Delta t$ are written as

$$
\begin{aligned}
& \Delta Q^{I}(t)=\theta(t) \cdot\left(I_{Q Q}(t)+I_{Q P}(t)\right) \cdot \Delta t, \\
& \Delta P^{I}(t)=\theta(t) \cdot\left(I_{P Q}(t)+I_{P P}(t)\right) \cdot \Delta t . \\
I_{Q Q}(t)= & B_{Q Q} \cdot V_{Q}(t), I_{Q P}(t)=B_{Q P} \cdot V_{P}(t), \\
I_{P Q}(t)= & B_{P Q} \cdot V_{Q}(t), I_{P P}(t)=B_{P P} \cdot V_{P}(t),
\end{aligned}
$$

- Investments made at time $t, B_{Q Q}, B_{Q P}, B_{P Q}, B_{P P}-$ norms of accumulation of internal endogenous investments.

Thus, formulas (4) become

$$
\begin{aligned}
& \Delta Q^{I}(t)=\theta(t) \cdot\left(B_{Q Q} \cdot V_{Q}(t)+B_{Q P} \cdot V_{P}(t)\right) \cdot \Delta t, \\
& \Delta P^{I}(t)=\theta(t) \cdot\left(B_{P Q} \cdot V_{Q}(t)+B_{P P} \cdot V_{P}(t)\right) \cdot \Delta t .
\end{aligned}
$$

The increments of external investments $\Delta Q^{G}(t)$, $\Delta P^{G}(t)$ for the period of time $\Delta t$ are determined by the relations.

$$
\begin{aligned}
& \Delta Q^{G}(t)=\theta(t) \cdot \eta_{Q} \cdot G(t) \cdot \Delta t, \\
& \Delta P^{G}(t)=\theta(t) \cdot \eta_{P} \cdot G(t) \cdot \Delta t .
\end{aligned}
$$

Here $G(t)$ is the total volume of external investment; $Q_{h}, h_{p}$, are the shares of the volumes of external investments $G(t)$ attributable to the volumes of factors of production $Q$ and $P$. It should be noted that the quantities, $h_{Q}, h_{P}$ are not independent, but satisfy the condition.

$$
\eta_{Q}+\eta_{P}=1
$$

Substitution of formulas (3), (5) and (6) into balance equations (2) gives.

$$
\begin{aligned}
& \Delta Q=\theta \cdot\left(-A_{Q} \cdot Q+B_{Q Q} \cdot V_{Q}+B_{Q P} \cdot V_{P}+\eta_{Q} \cdot G\right) \cdot \Delta t, \\
& \Delta P=\theta \cdot\left(-A_{P} \cdot P+B_{P Q} \cdot V_{Q}+B_{P P} \cdot V_{P}+\eta_{P} \cdot G\right) \cdot \Delta t .
\end{aligned}
$$

Substituting expressions for production functions into equations (7) and passing to the limit as $\Delta t \rightarrow 0$, we find a system of nonlinear differential equations.

$$
\begin{aligned}
& \frac{d Q}{d t}=\theta \cdot\left(-A_{Q} \cdot Q+B_{Q Q} \cdot R_{Q} \cdot Q^{a_{Q}}+B_{Q P} \cdot R_{P} \cdot P^{a_{P}}+\eta_{Q} \cdot G\right), \\
& \frac{d P}{d t}=\theta \cdot\left(-A_{P} \cdot P+B_{P Q} \cdot R_{Q} \cdot Q^{a_{Q}}+B_{P P} \cdot R_{P} \cdot P^{a_{P}}+\eta_{P} \cdot G\right) .
\end{aligned}
$$

Equations (8) form a system of normal nonlinear coupled equations of the first order, and its initial conditions are of the form.

$$
\begin{aligned}
& Q(0)=Q_{0}, \\
& P(0)=P_{0} .
\end{aligned}
$$

In the general case, the nonlinear Cauchy problem (8), (9) can be solved only numerically. The structure of balance equations (8) shows that the enterprise will develop as long as the volume of internal and external investments exceeds depreciation charges. The limit values $Q \infty$ and $P \infty$ of the volumes of production factors $Q(t)$ and $P(t)$ are found from equations.

$$
\begin{aligned}
& -A_{Q} \cdot Q+B_{Q Q} \cdot R_{Q} \cdot Q^{a_{Q}}+B_{Q P} \cdot R_{P} \cdot P^{a_{p}}+\eta_{Q} \cdot G=0, \\
& -A_{P} \cdot P+B_{P Q} \cdot R_{Q} \cdot Q^{a_{Q}}+B_{P P} \cdot R_{P} \cdot P^{a_{p}}+\eta_{P} \cdot G=0 .
\end{aligned}
$$


The function $\theta$ (t) describes the specific development rate of the enterprise. At a constant and unit speed $\theta(t)=1$, the development of the enterprise will be progressive and monotonically increasing. Various values of deviations of the function $\theta(t)$ from unity will correspond to a deceleration of the enterprise development process, its temporary stop during the change of production technologies and crisis phenomena. The forms of the integral curves of equation (8) substantially depend on the form of the function $\theta(t)$, which determines the center of the time interval, its length and the size of the deviation from the unit value at which the enterprise operates stably.

If in some temporary quarters with a center at a given point in time $\mathrm{t}=\mathrm{t} *$, the enterprise makes full or partial replacement of technological equipment, then the function $\theta(t)$ will have the form

$$
\theta(t)=1-(\omega) \cdot \exp \left(-\frac{\left(t-t^{*}\right)^{2}}{2 \cdot \sigma^{2}}\right)
$$

Here $\omega$ is the maximum size of the deviation of the function $\theta(t)$ from unity, $\sigma$ is the radius of the time interval $(\mathrm{t} *-\sigma, \mathrm{t} *+\sigma)$ on which the deviation of the function $\theta(\mathrm{t})$ from unity occurs.

In the process of modernization of the enterprise under consideration, the new production factor $\mathrm{P}$ gradually displaces the previous production factor $\mathrm{Q}$. At the same time, the value of output of old production VQ decreases to zero, and the limit value of output of new production VP is steadily increasing. Such a process can be described by the kinetic relation

$$
\mathbb{W}(t)=\mathbb{W}_{e}(t)+\mathbb{W}_{p}(t)=V_{e}(t) \cdot e^{-\lambda \cdot t}+V_{p}(t) \cdot\left(1-e^{-\lambda \cdot t}\right)
$$

Here, W (t) is the total value of the output of the enterprise as a whole; WQ ( $t$ ) and PW (t) are the values of output by manufactures when transferring old capacities to new ones.

\section{Results}

Figure 3 shows graphs of the production functions of each production component and the enterprise as a whole, constructed by the results of a numerical solution of the Cauchy problem (8), (9), which describe the process of replacing one production enterprise with another in the context of digital transformation of business processes $\omega=0$.

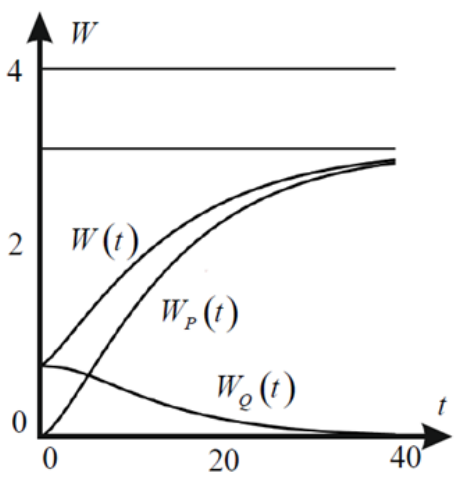

Figure 3. Graphs of production functions of each production component and the enterprise as a whole describing the process of replacing one production enterprise with another in the context of digitalization of business processes $\omega=0$

A numerical analysis of the model shows that in the case of an evolutionary modernization process, the maximum output of an enterprise occurs in that limited period of time when both manufactures work effectively in parallel. Then the old production is dismantled and the enterprise as a whole switches to a stable output in the new conditions.

Figure 4 shows the graphs of the production functions of each production component and the enterprise as a whole, constructed according to the results of a numerical solution of the Cauchy problem (8), (9), which describe the process of crowding out one production of an enterprise with another production, which is accompanied by a change in technological structures with a complete temporary shutdown of the enterprise $\omega=1, \mathrm{t} *=15$, $\sigma=5$.

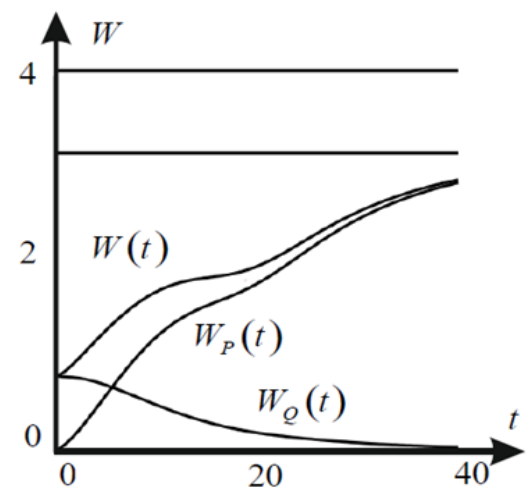

Figure 4. Graphs of production functions of each production component and the enterprise as a whole describing the process of crowding out one production of an enterprise with another production, which is accompanied by a change in technological structures with a complete temporary shutdown of the enterprise $\omega=1, t$

$$
*=15, \sigma=5
$$

A numerical analysis of the model in this case shows that in the case of a change in the technological structure of the enterprise, its maximum output occurs after a period of stagnation 
in a limited period of time, when the old production is still intensively operated. Then it is displaced, and the enterprise as a whole again switches to stable output in the new conditions.

Figure 5 shows graphs of the production functions of each production component and the enterprise as a whole, constructed by numerically solving the Cauchy problem (8), (9), which describe the process of crowding out one production of an enterprise with another production, which, in addition to changing technological structures, is accompanied by temporary crisis phenomena and elements temporary degradation of the enterprise $\omega=1,2 ; \mathrm{t} *=15 ; \sigma=5$.

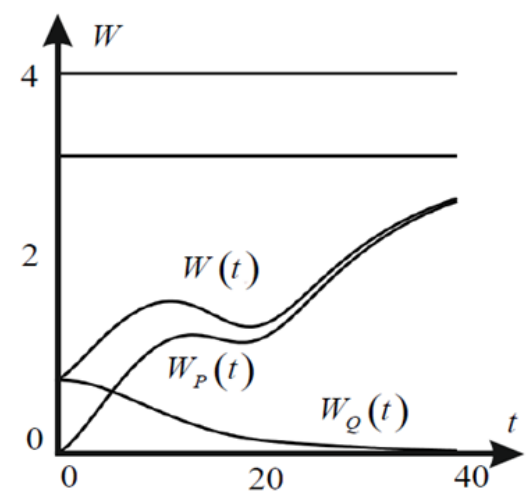

Figure 5. Graphs of production functions of each production component and the enterprise as a whole describing the process of crowding out one production of an enterprise with another production, which, in addition to changing technological patterns, is accompanied by temporary crisis phenomena and elements of temporary degradation of the enterprise $\omega=1,2 ; t *=15 ; \sigma=5$

Here, a numerical analysis of the model shows that in the case of a change in the technological structure of an enterprise accompanied by temporary crisis phenomena and elements of degradation, its maximum output occurs in a very limited period of time, after which the enterprise as a whole reaches a stable output in new conditions.

Estimated values: $R_{Q}=1,0 ; R_{P}=1,5 ; Q_{a}=0,35 ; a_{P}$ $=0,45 ; A_{Q}=0,20 ; A_{P}=0,12 ; B_{Q Q}=0,20 ; B_{Q P}=$ 0,$19 ; B_{P Q}=0,21 ; B_{P P}=0,18 ; \lambda=0,10$.

The resource limits $\mathrm{Q} \infty=4.6948$ and $\mathrm{P}_{\infty}=7.7067$ were calculated as a result of a numerical solution of the system of equations (10).

\section{Discussion}

Most companies now understand the need to digitize certain processes, but are not able to objectively evaluate first-rate steps and conduct an analytical and sociological study of the future implications for the company [1].

The survey, which comprises employees and business owners, show that the preparation for the digitalization is too long, which is explained by the lack of knowledge of the staff and the lack of experts and staff (Figure 6).
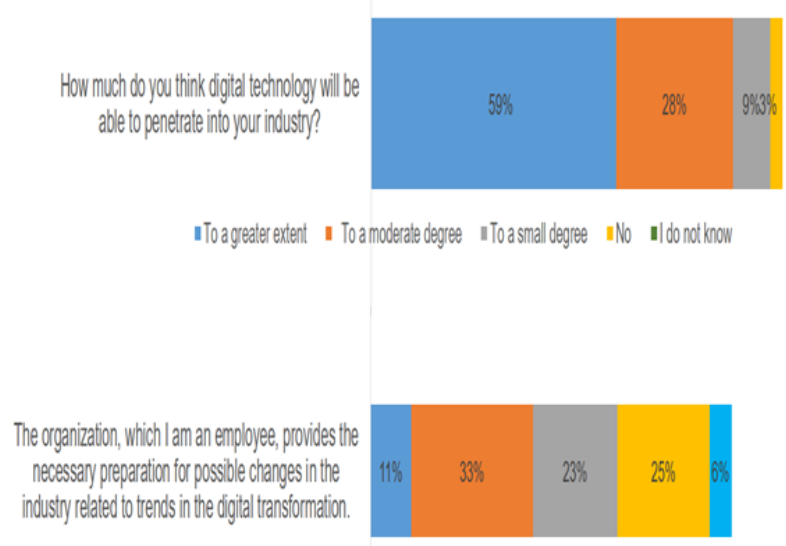

I|sirongly agree Ilagree I|can not give an answer IDisagree II stronglydisagree II don't know

Figure 6. The results of an in-house survey on the future level of digital penetration and the level of business preparation for this process

In addition, the use of individual digital technologies does not mean a digital transformation of the organization. The formation of an enterprise on the path to digital must necessarily go through the following steps (Figure 7):

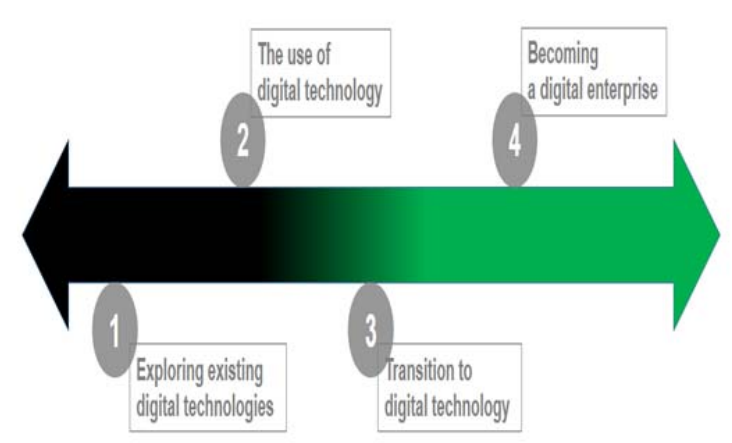

Figure 7. Stages of becoming a digital enterprise

At the same time, at the stage of studying digital technologies, traditional technologies are mobilized by attracting existing automation capabilities. Next, the most successful digitalization methods and tools are determined and digital technologies are applied. Then, after successful testing of the implemented digital technologies, they are introduced directly into the business processes of the enterprise. By gradually introducing business processes into the operating system and digital technologies in each operation, the enterprise becomes digital, which is, such an implementation provides an efficient and diverse use of operating models and business models for digitalization in future periods [11].

But leaders are now only at the stage of building up the necessary digital competencies and implementing of pilot projects. $89 \%$ of the largest industrial enterprises surveyed by KPMG said they 
started pilot projects or implemented solutions based on machine learning and artificial intelligence on a limited perimeter of processes. According to the OECD average, only $12 \%$ of enterprises in Western Europe already use big data analytics.

The digital transformation of business processes is a long process, and in order to bear fruit from the first steps, you need to "create a" road map "in accordance with the developed plans for technology implementation and changes in organizational processes.

This roadmap should be created jointly by specialists from different departments of the organization. The roadmap should help determine the basis of transformation, contain key objectives and justification of the structural elements of digital transformation in the enterprise (Figure 8).

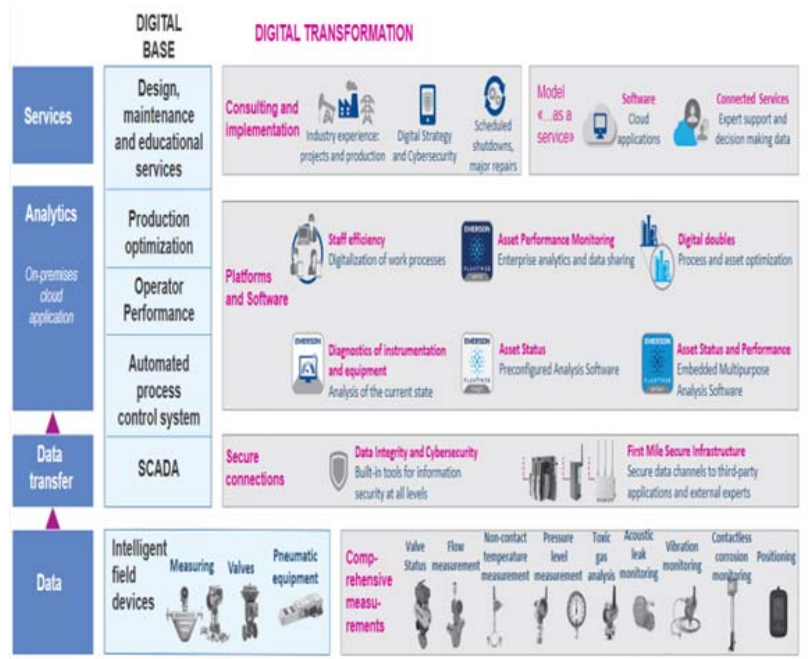

Figure 8. The basis, tasks and structural elements of digital transformation in the enterprise

The most important is the participation of the information technology department, which plays a decisive role in the determining of the solution architecture, and in the future will be responsible for the joint work of the implemented systems and programs.

Thus the serious role is played by the level of development of corporate culture in the enterprise, for the reason that digitalization of business is impossible without integration of innovations in the corporate culture. This is because, depending on the level of development of corporate culture at the enterprise, the speed of perception of innovation by all staff will occur [9]. There is also a correlation between the level of development of an enterprise, its time in the market and the timeliness of innovation implementation: the faster the organization develops, the more democratic the management style and the clearer the responsibilities shared between employees and the value of work for each employee based on collaboration, the easier it is to work with staff and convince them of the need for enterprise digital adoption (Figure 9).

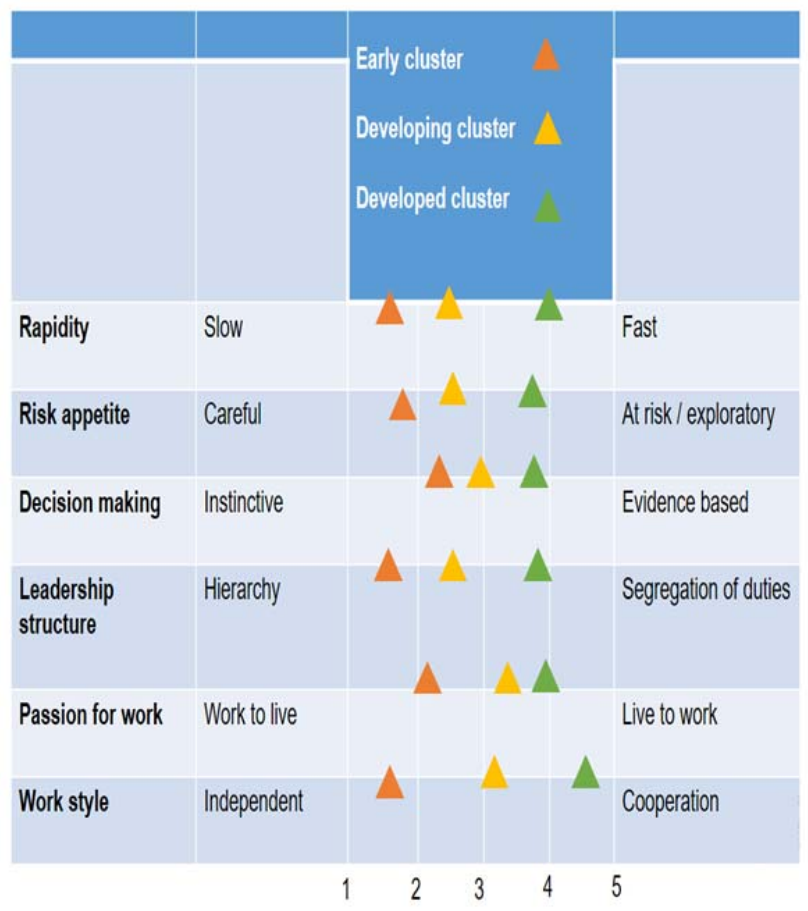

Figure 9. Integration of innovations in corporate culture

Solving of organizational issues is one of the most significant obstacles for digit al transformation business processes. In a recent Emerson survey, nearly two-thirds of digital technology and technology directors surveyed cited "different business ideas" as a major obstacle to the smooth operation of IT services and manufacturing units. At the same time, almost $80 \%$ of respondents noted that cooperation between these two areas is essential for a successful digital transformation. In general, this will allow us to move from the application of individual processes to the construction of a digital DNA code of an organization. The combination of innovation, flexibility and continuous updating throughout the digitalization of business processes will look as follows (Figure 10):

IT services and manufacturing units are responsible for safety and high efficiency. As part of business applications, IT professionals work with larger information systems and support the corporate data processing environment. Whether it is offices or industrial sites, such as power plants or offshore platforms, the IT service deals with functional and safety issues, supporting the system's uptime. They're masters of standards and scalability, said Peter Zornio, Chief Technology Officer, Emerson Automation Solutions. 


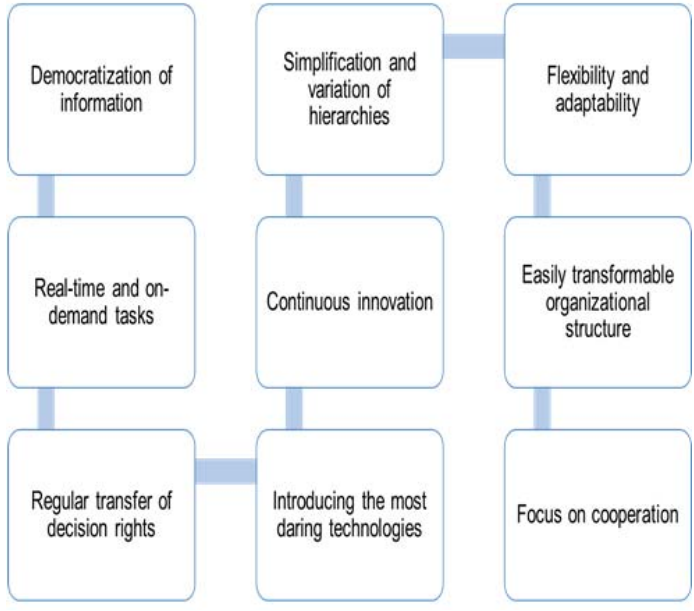

Figure 10. The combination of innovation, flexibility and continuous updating throughout the digital transformation of business processes

\section{Conclusions}

Companies that want to enter the digital zone have to do four things. First, they have to understand, in fact, what the value of digital transformation is in the automation of operations or a combination of all the constituent elements. Secondly, they need to prioritize. There is always too much to do in a digital portfolio, and it is important to prioritize. Thirdly,

\section{References}

[1]. Andriushchenko, K., Datsii, O., Aleinikova, O., Abdulla, A. M., \& Ali, A. M. (2019). Improvement of the water resources management system at the territorial level. Problems and Perspectives in Management, 17(3), 421. https://doi.org/10.21511.34

[2]. Baharun, R., Jing Mi, T., Streimikiene, D., Mardani, A., Shakeel, J., \& Nitsenko, V. (2019). Innovation in healthcare performance among private brand's healthcare services in small and medium-sized enterprises (SMEs). Acta Polytechnica Hungarica, 16(5), 151-172

https://doi.org/10.12700/APH.16.5.2019.5.9

[3]. Barrett, M., Davidson, E., Prabhu, J., \& Vargo, S. L. (2015). Service innovation in the digital age: key contributions and future directions. MIS quarterly, 39(1), 135-154.

[4]. Brynjolfsson, E., \& McAfee, A. (2014). The second machine age: Work, progress, and prosperity in a time of brilliant technologies. WW Norton \& Company.

[5]. Christensen, C. M. (2013). The innovator's dilemma: when new technologies cause great firms to fail. Harvard Business Review Press.

[6]. Dougherty, D., \& Dunne, D. D. (2012). Digital science and knowledge boundaries in complex innovation. Organization Science, 23(5), 1467-1484. http://doi.org/10.1287/orsc. 1110.0700 they have to have an end-to-end view to ensure that customers get combined experience from end to end and that all functions work together. And finally, they need to look at their portfolio of companies and understand what impact digital technologies can have on valuations, and they should focus on what necessary or required opportunities will develop, and possibly balance the portfolio accordingly.

Perhaps the most difficult task for organizations is collaborative and integrated work. Many organizations are distributed by various functions or geographical regions. But digital customers expect a completely consistent and unified experience. And this requires companies to have a completely different approach to the organization, their management structures and standards for data and systems.

The art of managing companies in the context of introducing a new business process is to harmonize the business with digital transformations. This serves as a guide for demonstrating the potential of the business process being implemented. The company's management should adequately assess its potential in terms of identifying approaches that use both internal and external resources. After this, finally, the stage of actual implementation of the business process begins.

[7]. Frank, M., Roehrig, P., \& Pring, B. (2014). Code halos: How the digital lives of people, things, and organizations are changing the rules of business. John Wiley \& Sons.

[8]. Rivera, J., \& van der Meulen, R. (2014). Gartner says 4.9 billion connected 'things' will be in use in 2015. Gartner.

[9]. Google Trends. (2019). Digital Transformation. Retrieved from: https://trends.google.com/trends/explore? $\mathrm{q}=$ digital $\% 2$ 0transformation [accessed: 16 August 2020].

[10]. Havrysh, V., Nitsenko, V., Bilan, Y., \& Streimikiene, D. (2019). Assessment of optimal location for a centralized biogas upgrading facility. Energy \& Environment, 30(3), 462-480. https://doi.org/10.1177/0958305X18793110.

[11]. Kane, G. C., Palmer, D., Phillips, A. N., \& Kiron, D. (2015). Is your business ready for a digital future?. MIT Sloan management review, 56(4), 37-44.

[12]. Keen, P., \& Williams, R. (2013). Value architectures for digital business: beyond the business model. Mis Quarterly, 37(2), 643-647.

[13]. Liezina, A., Andriushchenko, K., Rozhko, O., Datsii, O., Mishchenko, L., \& Cherniaieva, O. (2020). Resource planning for risk diversification in the formation of a digital twin enterprise. Accounting, 6(7), 1337-1344. https://doi.org/10.5267/j.ac.2020.8.016 
[14]. Lyytinen, K., Yoo, Y., \& Boland Jr, R. J. (2016). Digital product innovation within four classes of innovation networks. Information Systems Journal, 26(1), 47-75. https://doi.org/10.1111/isj.12093.

[15]. Markides, C., \& Oyon, D. (2010). What to do against disruptive business models (when and how to play two games at once). MIT Sloan Management Review, 51(4), 25.

[16]. Markides, C. C. (2013). Business model innovation: what can the ambidexterity literature teach us?. Academy of Management Perspectives, 27(4), 313-323.

[17]. Parker B., Baya V., \& Morrison A. (2012). Technology forecast - The business value of APIs. PwC Technology Forecast: Rethinking integration, pp.1-76.
[18]. Vogelsang, M. (2010). Digitalization in open economies: Theory and policy implications. Springer Science \& Business Media.

[19]. Westerman, G., Bonnet, D., \& McAfee, A. (2014). Leading digital: Turning technology into business transformation. Harvard Business Press.

[20]. Yoo, Y., Henfridsson, O., \& Lyytinen, K. (2010). Research commentary - the new organizing logic of digital innovation: an agenda for information systems research. Information systems research, 21(4), 724735.

[21]. Yu, L. (2006). Understanding information inequality: Making sense of the literature of the information and digital divides. Journal of Librarianship and Information Science, 38(4), 229-252. 